Hydroécol. Appl. (1991) 1, pp. 1 - 26

\title{
Utilisation des rejets d'eaux tièdes des centrales thermiques en aquaculture
}

\author{
G. Merle \\ Electricité de France \\ Direction de l'Equipement 22-30, avenue de Wagram 75008 PARIS
}

Résumé. - L'utilisation des eaux tièdes rejetées par les centrales thermiques est possible en aquaculture. Des expérimentations et essais menées sur sites pendant de nombreuses années ont permis d'en cerner les avantages et les inconvénients. Des élevages industriels fonctionnent maintenant avec succès à Gravelines et de nombreuses informations ont été recueillies sur des installations-pilotes. Elles sont disponibles pour aider à la mise en place de nouvelles unités sur les sites où la fourniture de l'eau tiède aux installations d'aquaculture pourra être réalisée à un coût économiquement supportable.

Summary. - Heated water from power plants can be used for aquaculture applications. Experiments and demonstrations on power plant sites during many years have shown the inconvenients and benefits from the use of warmed water. Some fish farms operate now on an industrial scale at Gravelines (59). Pilot experiments opened the way for new creations on the sites where facilities using heated water can be find.

\section{INTRODUCTION}

L'idée d'utiliser les rejets d'eaux tièdes en provenance des centrales thermiques remonte maintenant à une quinzaine d'années. Les premières réflexions ont été engagées avec le Commissariat de l'Energie Atomique vers 1970 et se sont intensifiées, après le choc pétrolier de 1972-1973, dans une préoccupation générale d'économie d'énergie et de meilleure insertion des centrales dans leur environnement.

L'échauffement subi par les eaux prélevées dans le milieu naturel, fleuve ou mer, est relativement faible et la température finale des eaux tièdes est trop basse pour envisager une utilisation secondaire dans des processus industriels. Aussi s'est-on très tôt tourné vers les processus biologiques. Pour ceux-ci l'influence bénéfique d'une élévation de tempé- 
G. Merle

rature est bien connue, à condition toutefois de ne pas dépasser certaines températures limites, ellesmêmes relativement basses. Des applications ont donc été recherchées à la tois dans le domaine agricole et le domaine aquacole. Après une phase d'expérimentations et de démonstrations de plusieurs années, on assiste maintenant à l'installation progressive de professionnels de l'horticulture, du maraîchage et de l'aquaculture autour de plusieurs centrales nucléaires.

Nous nous proposons, dans cet article, de présenter les connaissances acquises en matière d'aquaculture en eaux tièdes et d'aborder les perspectives de développement compte tenu des difficultés précédemment rencontrées.

\section{1. - LES CONDITIONS D'UTILISATION DES REJETS THERMIQUES}

\subsection{Avantages et Inconvénients d'une augmentation de la température}

Le principal intérêt des eaux tièdes rejetées par les centrales thermiques pour l'aquaculture réside dans le fait qu'elles peuvent contribuer à l'accélération des phénomènes de croissance des organismes aquatiques. En effet, pour la majorité des animaux aquatiques (Poissons, Crustacés, Mollusques...) l'ensemble des processus métaboliques est affecté directement par le niveau de température du milieu. En stimulant à la base les ac-

Tableau 1 - Effets d'une augmentation de la température du milieu d'élevage des poissons d'après Duret et al. (1989).

\begin{tabular}{|c|c|}
\hline $\begin{array}{c}\text { Effets } \\
\text { favorables }\end{array}$ & $\begin{array}{l}\text { Augmentation globale des métabolismes } \\
\text { d'ou: } \\
\text { - Diminution de la duree de maturation } \\
\text { - Extension de la periode de ponte } \\
\text { - Acceleration de la croissance a tous les stades } \\
\text { - Diminution des indices de consommation } \\
\text {-... }\end{array}$ \\
\hline $\begin{array}{c}\text { Effets } \\
\text { defavorables }\end{array}$ & $\begin{array}{l}\text { Diminution de la solubilité de l'oxygene dans l'eau } \\
\text { Augmentation de la toxicite de certains } \\
\text { rejets (ex: Ammoniac en milieu basique) } \\
\text { Accélération du développement de } \\
\text { certains germes pathogènes (ex : bactéries) } \\
\text { Augmentation de la consommation } \\
\text { d'oxygène (due a l'augmentation du métabolisme) }\end{array}$ \\
\hline
\end{tabular}


tivités enzymatiques, l'élévation de la température accélère la croissance des animaux dans les limites propres à chaque espèce. Cela ne va pas sans quelques effets défavorables sur le milieu d'élevage (Tableau I) qu'il convient de prendre en compte.

Pour les espèces des climats tempérés, la possibilité d'utiliser une eau d'élevage dont la température est proche de l'optimum thermique de l'espèce considérée, se traduit concrètement pour l'aquaculteur par une diminution de la durée moyenne du cycle d'élevage d'où une rotation accélérée des stocks et une meilleure utilisation des infrastructures conduisant à une réduction des coûts de production. On peut ainsi obtenir un silure glane (Silurus glanis) de $1,5 \mathrm{~kg}$ en 15 mois ou un esturgeon sibérien (Acipenser baeri) de $2,5 \mathrm{~kg}$ en 2 ans, soit un gain de temps de 6 à 12 mois par rapport à des élevages en températures naturelles.

Mais il est également possible d'utiliser les eaux tièdes pour étendre les zones traditionnelles d'élevage d'une espèce donnée. C'est le cas à Gravelines dans le Nord de la France où les rejets de la centrale permettent de reproduire les conditions thermiques de la Méditerranée nécessaires à l'élevage du bar (Dicentrachus labrax).

Cela permet même l'élevage d'espèces tropicales dans la perspective

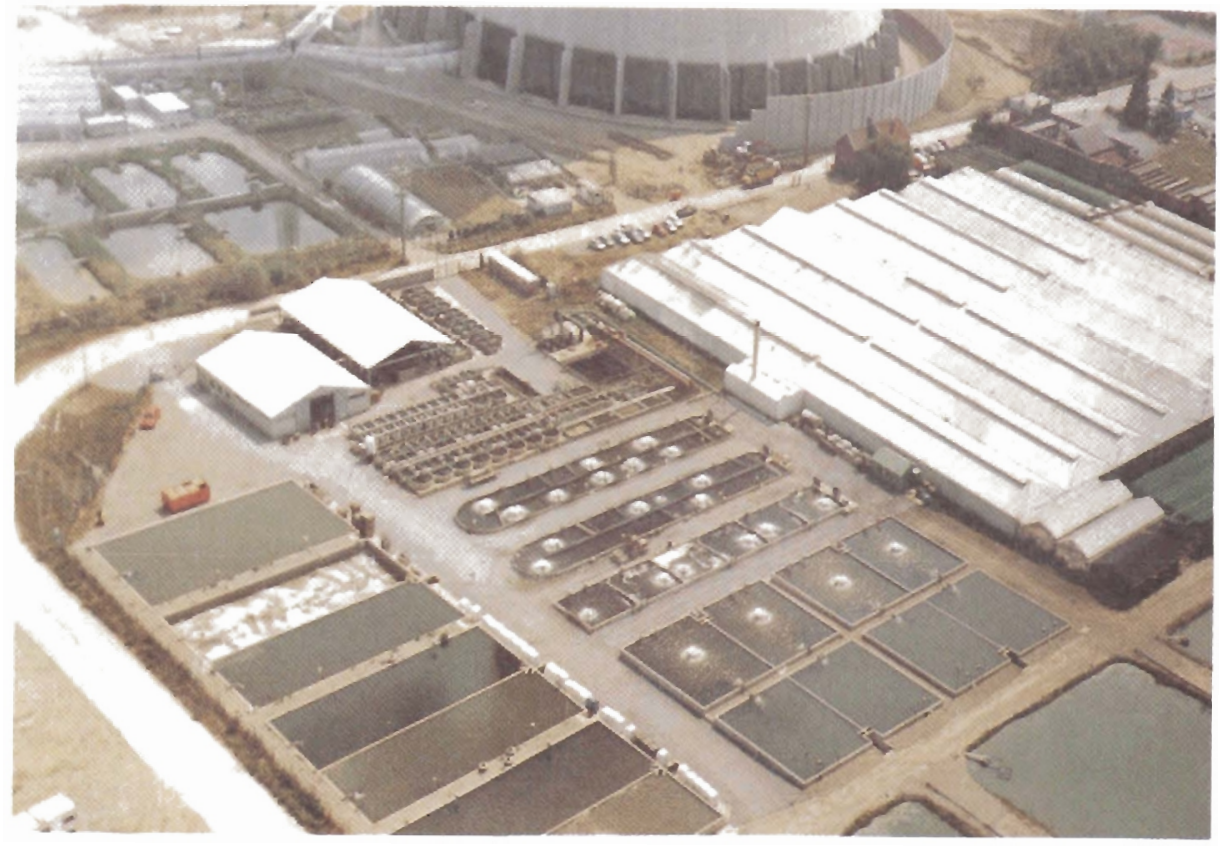

Photo 1 - Piscimeuse S.A. : Pisciculture intensive de tilapias, carpes et poissons-chat à Thange (Belgique). 
d'une diversification des productions. Dans ce cadre l'espèce qui a fait l'objet de nombreuses expérimentations en France, en Italie et en Belgique est le tilapia. Actuellement la production de tilapias est réalisée de façon industrielle sur le site de TIHANGE en Belgique (Photo 1). Plus de 200 tonnes de tilapias appartenant aux deux espèces Oreochromis niloticus (Tilapias du Nil) et Oreochromis aureus (Tilapia doré) sont produites chaque année (Philippart et al., 1989).

Enfin l'élévation de la température de l'eau permet de s'affranchir en partie de l'effet des saisons et d'obtenir des pontes décalées et un grossissement précoce des jeunes individus. Ceci peut être mis à profit dans les écloseries et unités de pré-grossissement même si celles-ci utilisent des débits d'eau moins importants que des unités de grossissement. C'est le cas à Gravelines dans l'Ecloserie Marine où l'on produit des alevins de bars, de daurades et récemment (1990) de turbots.

\subsection{Cadre législatif et conditions de fourniture des eaux tièdes}

Comme nous l'avons précisé en introduction, Electricité de France a étudié dès 1975, pour chacune de ses centrales en construction, la possibilité de prélever sur ses installations des effluents thermiques afin de les mettre à la disposition d'utilisateurs intéressés et a mis en place des expérimentations sur certains sites. En 1980 , cette volonté a été confirmée dans la loi $n^{\circ} 80-531$ du 15 juillet 1980 relative aux économies d'éner- gie et à l'utilisation de la chaleur qui définit aussi le rôle des collectivités locales dans la promotion et le développement des réseaux de chaleur. Les exploitants des unités thermiques de production d'électricité doivent favoriser la création et le développement des réseaux de chaleur, en accord avec les collectivités locales.

Le décret du 13 mai 1981, pris en application de cette loi, précise en particulier que la chaleur provenant des rejets thermiques est gratuite mais que les charges d'équipement, d'entretien, de renouvellement et d'exploitation des installations de récupération sont à la charge de l'utilisateur.

A ceci s'ajoute le fait que la fourniture d'eau tiède ne peut être garantie ni en débit, ni en température car elle est liée au fonctionnement des unités. Elle peut être interrompue lors des arrêts de la centrale pour des raisons techniques comme le rechargement, les inspections, l'entretien... ou économiques. C'est pourquoi le raccordement à plusieurs unités d'un même site est nécessaire pour améliorer la disponibilité en eau. Malgré cela on s'assurera que l'utilisateur de chaleur dispose d'installations de secours ou de moyens palliatifs pour protéger sa production quelle que soit la durée d'interruption de la fourniture d'eau tiède.

\subsection{Disponibilités en eau}

Les quantités d'eau tiède disponibles sont très différentes selon le mode de réfrigération mis en œuvre sur un site donné. 
- Pour une centrale refroidie en circuit ouvert ce qui est le cas lorsqu'on dispose de grandes quantités d'eau par exemple en bord de mer ou sur un grand fleuve le débit pompé pour assurer le refroidissement est d'environ $40 \mathrm{~m}^{3} / \mathrm{s}$ pour une unité de production d'électricité de $900 \mathrm{MWe}$ et de $45 \mathrm{~m}^{3} / \mathrm{s}$ pour une unité de $1300 \mathrm{MWe}$. Comme il y a de 2 à 6 unités par site, cela représente des débits importants, théoriquement disponibles pour l'aquaculture. Les limites au prélèvement d'importants volumes d'eau sur de tels sites sont uniquement d'ordre technique et économique, liés aux problèmes de pompage et de transport. C'est ainsi que les calculs économiques effectués conduisent à envisager des prélèvements d'eau de 3 à $20 \mathrm{~m}^{3} / \mathrm{s}$ permettant d'alimenter 2 a 15 ha de bassins aquacoles (Figure 1).

L'eau tiède est rejetée dans le milieu naturel avec une élévation de température à peu près constante de 10 à $15^{\circ} \mathrm{C}$ selon la puissance produite par l'unité. A élévation constante, les eaux tièdes suivent les variations de la température naturelle de l'eau (Figure 3 - courbes 2 et 3 ). A certaines périodes de l'année (surtout l'été) les températures atteintes par les eaux tièdes ne seront pas favorables à l'élevage. II est donc nécessaire de disposer sur le site retenu pour l'aquaculture, d'une possibilité de prélever de l'eau froide directe-

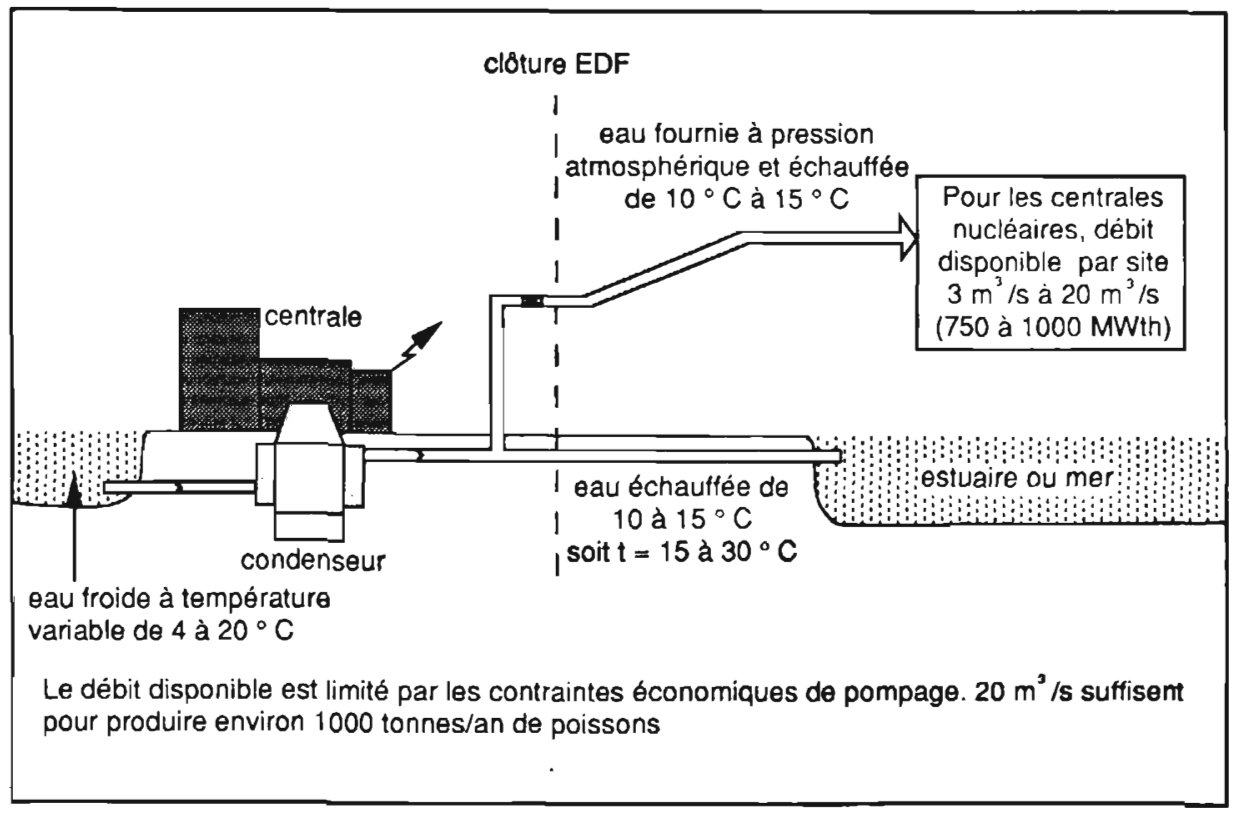

Fig. 1 - Schéma de prélèvement sur un circuit ouvert (fleuve à gros débit, estuaire ou bord de mer). 
ment dans la mer ou le fleuve, soit pour pratiquer un mélange avec les eaux réchauffées, soit pour remplacer celles-ci en période estivale. Ceci permet de "régler" en permanence la température d'élevage au niveau optimal de croissance des espèces retenues. C'est un des avantages majeurs des centrales thermiques même si cela nécessite la mise en place onéreuse d'un double réseau de canalisations.

- Dans une centrale refroidie en circuit fermé, les calories ne sont pas rejetées directement dans l'eau, mais à l'atmosphère par l'intermédiaire d'un réfrigérant atmosphérique. $\mathrm{Ce}$ mode de réfrigération est utilisé dans les centrales situées très en amont sur les fleuves ou sur des cours d'eau à débit moyen pour lesquels les ressources en eau sont limitées car il réduit considérablement les débits pompés puis rejetés échauffés dans la rivière. Pour une unité de $1300 \mathrm{MWe}$, le débit d'eau prélevé dans le cours d'eau sera seulement d'environ 2 à $3 \mathrm{~m}^{3} / \mathrm{s}$ destinés à compenser l'évaporation dans le réfrigérant et à pratiquer une purge pour éviter une concentration des sels minéraux dans le circuit.

Dans ce type de circuit, l'eau tiède est prélevée sur la "branche chaude" du circuit c'est-à-dire après le condenseur et avant l'arrivée dans le réfrigérant atmosphérique. Compte tenu des spécificités techniques des réfri-

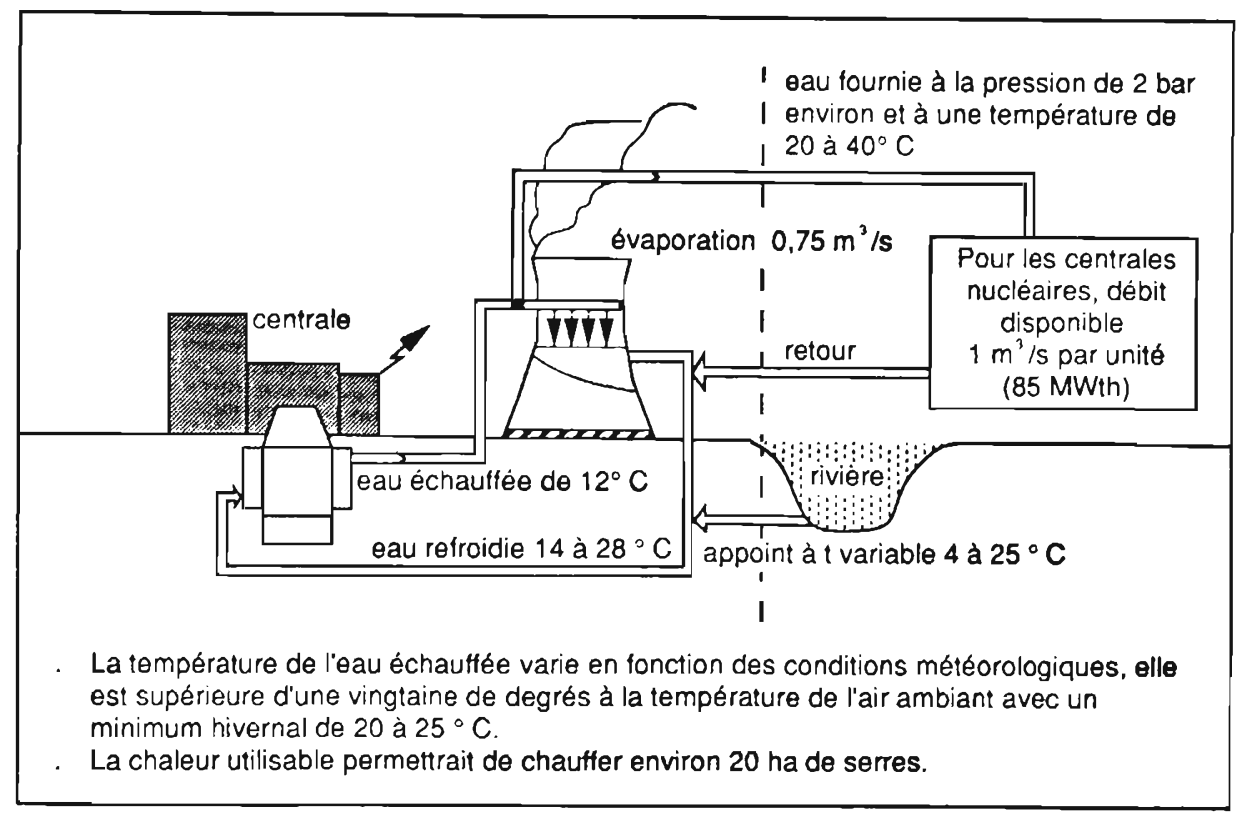

Fig. 2 - Schéma de prélèvement sur un circuit fermé (rivière à faible débit). 
gérants et notamment du facteur de concentration le débit maximal disponible est de $1 \mathrm{~m}^{3} / \mathrm{s}$ par unité. L'eau est fournie à une pression d'environ 2 bar ce qui permet une desserte gravitaire des installations selon leur localisation. Après utilisation l'eau doit être restituée pour respecter les autorisations administratives de prise et rejet d'eau, mais le point de retour peut être légèrement différent selon les sites (Figure 2).

Le température de l'eau tiède disponible est supérieure d'une vingtaine de degrés à la température de l'air ambiant avec un minimum de l'ordre de 20 à $25^{\circ} \mathrm{C}$ en période hivernale et un maximum de 40 à $45^{\circ} \mathrm{C}$ en été (Figure 3 - courbes 1 et 4 ).

\subsection{Qualité physico-chimique des eaux tièdes}

L'élévation rapide de la température dans les condenseurs a pour conséquence de diminuer la solubilité des gaz dans l'eau. II se produit alors une sursaturation qui peut persister quelque temps car le dégazage naturel se fait lentement (Hussenot et al., 1987).

La sursaturation en gaz dissous, surtout en azote, doit être évitée en aquaculture. Au-dessus d'un seuil de $105 \%$ (pression en gaz totaux exprimée en pourcentage de la pression barométrique) les poissons présentent les signes cliniques de la «maladie de la bulle" (bubble gas disease) qui peut entraîner de fortes

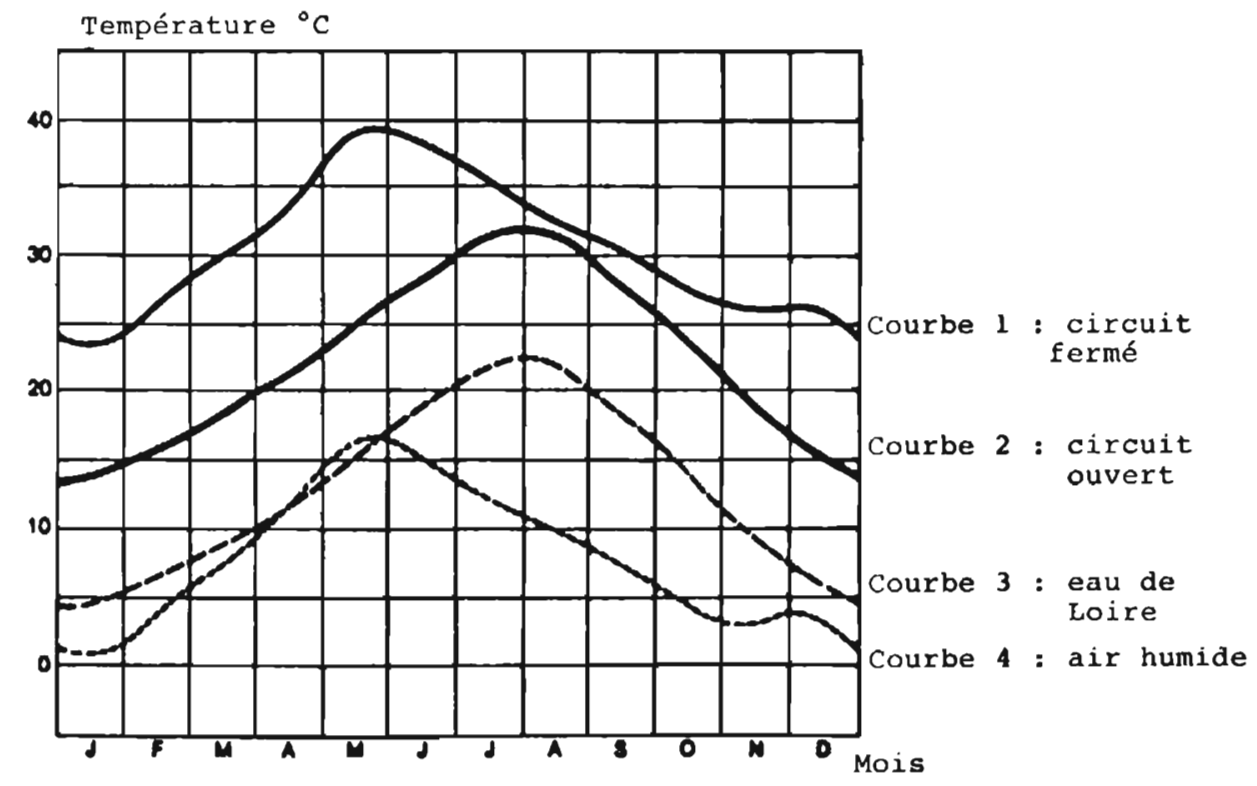

Fig. 3 - Evolution de la température des eaux de refroidissement. Exemple : Saint-Laurent-desEaux sur la Loire. 
mortalités. II sera donc nécessaire de prévoir un traitement des eaux tièdes en entrée de pisciculture pour contrôler le taux de sursaturation à l'aide de matériels tel que dégazeurs, aérateurs ou colonnes de dégazage...

D'autre part certains produits chimiques sont injectés dans les circuits de refroidissement afin de préserver le pouvoir d'échange des condenseurs. Une chloration à faible dose est utilisée dans les centrales du bord de mer pour lutter contre la fixation des moules dans les circuits d'eau. Les quantités de chlore résiduel dans les eaux rejetées sont minimisées en permanence mais il est préférable de disposer à l'entrée de la pisciculture d'un dispositif d'analyse en continu de la teneur en chlore couplé avec un système injectant un neutralisant du chlore, si les quantités en chlore résiduel dépasse le seuil toxique pour les poissons.

Dans les centrales fonctionnant en circuit fermé, un traitement peut être utilisé pour éviter l'entartrage. II s'agit le plus souvent d'injections d'acide sulfurique qui conduisent à une légère augmentation de la teneur en sulfates des eaux sans conséquence pour un élevage aquacole.

Enfin, il peut être nécessaire de réaliser une chloration massive pour réduire la croissance des algues dans les tours des réfrigérants atmosphériques. Cette opération, très rare et de courte durée, s'opère en suspendant l'alimentation de la zone utilisatrice des eaux tièdes par l'unité concernée par le traitement.

\subsection{Sites disponibles}

Tous les sites classiques ou nucléaires existants aujourd'hui sont théoriquement susceptibles de fournir des eaux tièdes à d'éventuels utilisateurs. Pourtant, si l'on se réfère à la carte de la Figure 4, il apparaît qu'il n'y a que sept sites qui possèdent un réseau de distribution d'eau tiède: Gravelines, Chinon, St Laurent, Dampierre, Bugey, Cruas et le Blayais, dont deux, Gravelines et le Blayais, refroidis en circuit ouvert, bénéficient d'une valorisation des eaux échauffées par l'aquaculture. Sur les 5 autres sites, les eaux tièdes sont utilisés en horticulture et maraîchage (E.D.F., 1990). Ceci résulte de nombreux facteurs parmi lesquels on peut citer:

\section{- le positionnement géographique}

Près des centrales côtières, l'eau de mer est disponible en grandes quantités, mais sur les côtes à falaises, les centrales ont été réalisées dans des excavations ce qui ne laisse que peu, voire pas d'espace disponible pour installer, au niveau de la centrale, des bassins d'aquaculture. C'est le cas à Paluel, Penly et Flamanville.

A Belleville, sur la Loire, c'est la présence de zones inondables autour de la centrale qui pose le même problème. Sur d'autres sites, le coût des ouvrages pour relier les terrains disponibles à la centrale est prohibitif (exemple : Nogent-sur-Seine). 


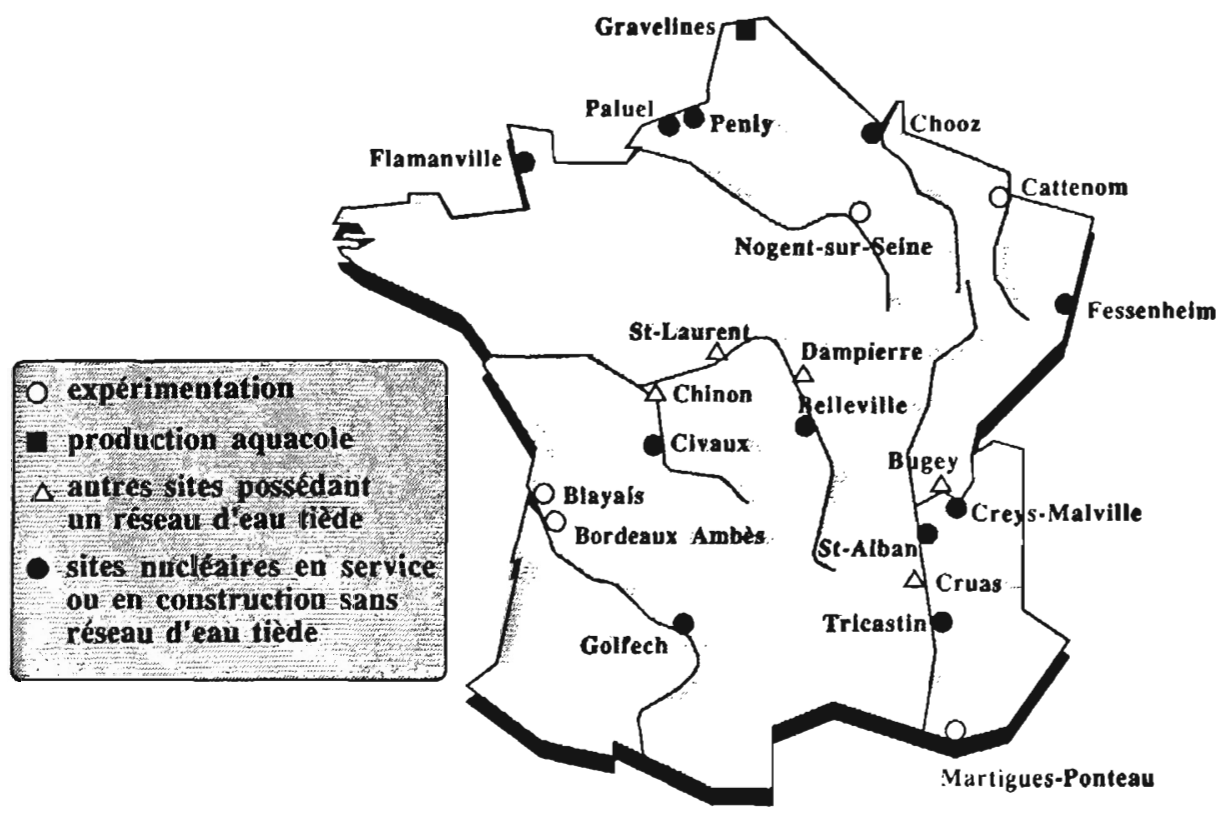

Flg. 4 - Sites concernés par l'aquaculture en eaux tièdes.

\section{- le mode de refroidissement}

L'aquaculture est en général grosse consommatrice d'eau et l'on s'est d'abord attaché à utiliser les eaux tièdes fournies par les circuits ouverts dont les températures sont assez proches des températures optimales de croissance des poissons.

Pour les circuits fermés où les débits disponibles sont faibles il faut disposer d'une technologie d'élevage plus sophistiquée faisant appel à un recyclage partiel de l'eau et d'une source d'eau froide abondante et de bonne qualité, d'autant plus que la température de l'eau tiède est la majeure partie du temps trop élevée. C'est un problème que l'on doit ré- soudre à Cattenom pour pouvoir développer l'aquaculture.

\section{- le nombre d'unités sur un}

même site

Une caractéristique importante à prendre en compte est le risque d'interruption de la fourniture d'eau tiède. Le branchement sur plusieurs unités permet d'améliorer la disponibilité de la fourniture. Avec un raccordement sur deux unités, des risques d'interruption pendant une durée supérieure à une semaine existent tandis qu'avec un prélèvement sur 4 unités la disponibilité est quasi-totale, c'est-àdire que le risque d'arrêt simultané des 4 unités est extrêmement faible. 
- enfin la qualité de l'eau avant échauffement doit être bonne ce qui n'est pas toujours le cas avec les pollutions chroniques ou insidieuses qui touchent les fleuves français notamment le Rhône. Ceci a été l'une des causes de l'abandon d'un projet d'aquaculture au Bugey.

Tableau 2 - Caractéristiques des essais d'aquaculture en eau tiède.

\begin{tabular}{|c|c|}
\hline SITES & NATUAE DES ELEVAGES \\
\hline \multicolumn{2}{|c|}{ ANCIENS SITES EXPERIMENTAUX } \\
\hline $\begin{array}{l}\text { Bordeaux Ambes (33) } \\
\text { (eau legerement sau- } \\
\text { matre) }\end{array}$ & $\begin{array}{l}\text { Eaux tieddes en provenance d'un circuit ouvert - } \\
\text { Etudes experimentales sur carpes, truites, an- } \\
\text { guilles, silures, esturgeons, tilapias de } 1976 \text { a } 1979 .\end{array}$ \\
\hline $\begin{array}{l}\text { St-Laurent des Eaux (41) } \\
\text { (eau douce) }\end{array}$ & $\begin{array}{l}\text { Eaux tieddes on provenance d'un circuit ouvert (St-Laurent } \\
\text { A) ot ferme (St-Laurent B), expérimentations sur le brochet, } \\
\text { l'anguille, les tilapias et les poissons d'ornement. Fin des } \\
\text { essais en } 1986 \text {. }\end{array}$ \\
\hline $\begin{array}{l}\text { Martigues-Ponteau (13) } \\
\text { (eau de mer) }\end{array}$ & $\begin{array}{l}\text { Eaux tiedes en provenance d'un circuit ouvert - Essais sur le } \\
\text { bar, la daurade, les crustacés et les tortues de } 1975 \text { a } 1979 . \\
\text { Ecloserie de bars et de daurades de } 1980 \text { a } 1987 .\end{array}$ \\
\hline $\begin{array}{l}\text { Nogent-sur-Seine (10) } \\
\text { (eau douce) }\end{array}$ & $\begin{array}{l}\text { Simulation d'eaux tièdes en provenance d'un circuit fermé. } \\
\text { Etude expérimentale d'elevage d'anguilles de } 1986 \text { à } 1988 .\end{array}$ \\
\hline $\begin{array}{l}\text { Le Blayais (83) } \\
\text { (eau saumatre) }\end{array}$ & $\begin{array}{l}\text { Eaux tieddes en provenance d'un circuit ouvert - Essais sur } \\
\text { bars, anguilles, daurades de } 1982 \text { a } 1985 \text {. De } 1985 \text { a } 1990, \\
\text { grossissement de bars et d'esturgeons. Plus de } 5 \text { tonnes de } \\
\text { biomasse en place essentiellement en esturgeons. }\end{array}$ \\
\hline \multicolumn{2}{|c|}{ SITES EXPERIMENTAUX EN FONCTIONNEMENT } \\
\hline $\begin{array}{l}\text { Cattenom }(5 \pi) \\
\text { (eau douce) }\end{array}$ & $\begin{array}{l}\text { Eau de la retenue du Mirgenbach legèrement bchauffé par } \\
\text { purges des réfrigérants. Essais en cages flottantes sur } \\
\text { carpes depuis } 1989 \text {. }\end{array}$ \\
\hline
\end{tabular}




\section{2. - L'EXPÉRIENCE ACQUISE} DEPUIS 1975

A partir de 1975-1976 trois sites de centrales ont été équipés afin d'étudier les possibilités de l'aquaculture en eau tiède dans les différents milieux, marin, dulçaquicole et saumâtre.

Les caractéristiques principales de ces 3 sites sont résumées sur le Tableau II.

\section{Martigues-Ponteau}

Sur cette centrale, la seule du littoral méditerranéen, les expériences ont d'abord été menées par le CE-
MAGREF. C'était la première tentative de ce genre réalisée en France. En se basant sur les résultats positifs obtenus, E.D.F. a encouragé, en 1979, la création de Delta-Aquaculture, association qui a confié l'exploitation de la station à Sepia-International, société d'ingénierie et de production aquacole. L'installation est alors devenue pendant sept ans un des principaux sites français d'écloserie et de prégrossissement de bars et de daurades avec une production annuelle voisine de 400000 alevins (Photo 2). L'installation n'a cessé de fonctionner qu'en 1987 lors de la mise en service de l'Ecloserie Marine de Gravelines qui en est, en quelque sorte, la grande sœur.

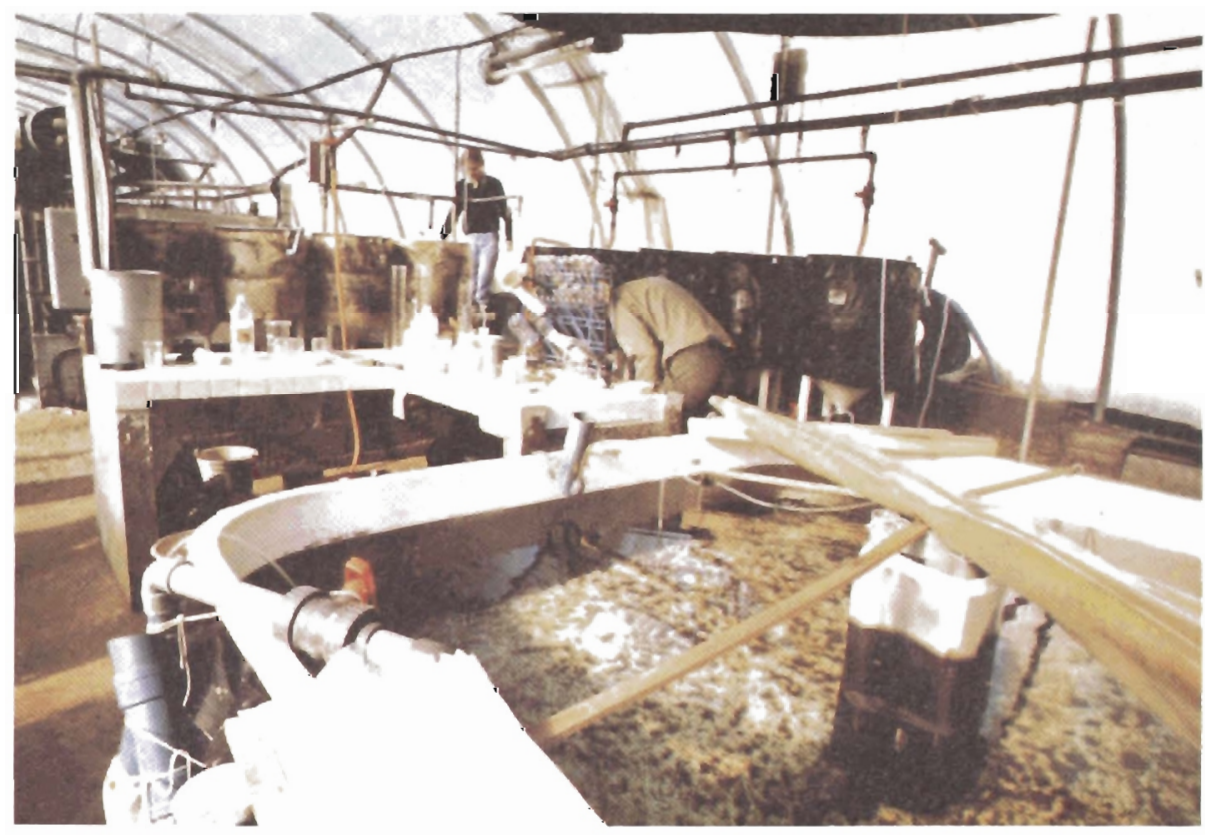

Photo 2 - Station d'écloserie et de progrossissement de bars el de daurades installée dans la centrale de Martigues-Ponteau (13). 


\section{Bordeaux-Ambés}

Sur cette centrale située en aval de Bordeaux sur l'estuaire de la Gironde les expérimentations menées par le CEMAGREF, en portant sur un grand nombre d'espèces, ont permis d'opérer une pré-sélection d'espèces, dont les performances en eaux tièdes étaient intéressantes. Ceci a permis d'orienter le choix des espèces qui ont été ensuite élevées sur le pilote installé sur le site du Blayais.

\section{St-Laurent des Eaux}

La station d'aquaculture de St-Laurent des Eaux a fonctionné de 1976 à 1986 (Photos 3 el 4). L'objectif pour- suivi lors des deux premières années de fonctionnement était la production de brochetons de repeuplement, mais cette activité ne nécessitait pas le recours à de grandes quantités d'eau tiède. II a donc semblé préférable de s'orienter, à partir de 1978, vers une démonstration de production commerciale d'anguilles. L'objectif était un pilote commercial produisant 30 à 50 t/an d'anguilles d'un poids moyen de $200 \mathrm{~g}$. De mauvaises conditions d'alimentation en eau, alliées à de grandes difficultés pour maîtriser les paramètres zootechniques et la pathologie dans un élevage d'anguilles, n'ont pas permis d'atteindre les résultats escomptés. Les essais

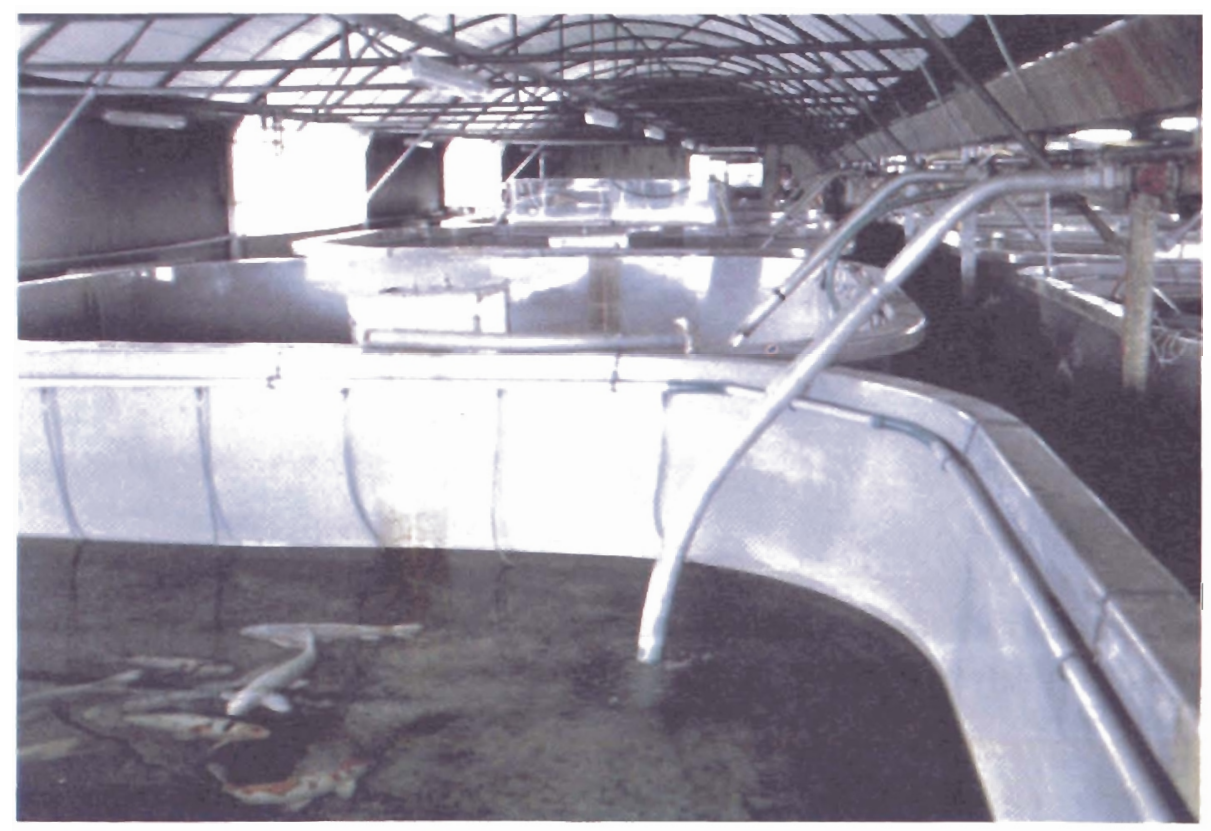

Photo 3 - Station d'aquaculture de Saint-Laurent des Eaux (41).

Bassins sub-carrés sous abris couverts, utilisés pour l'élevage des anguilles, puis pour la reproduction des poissons d'ornement. 


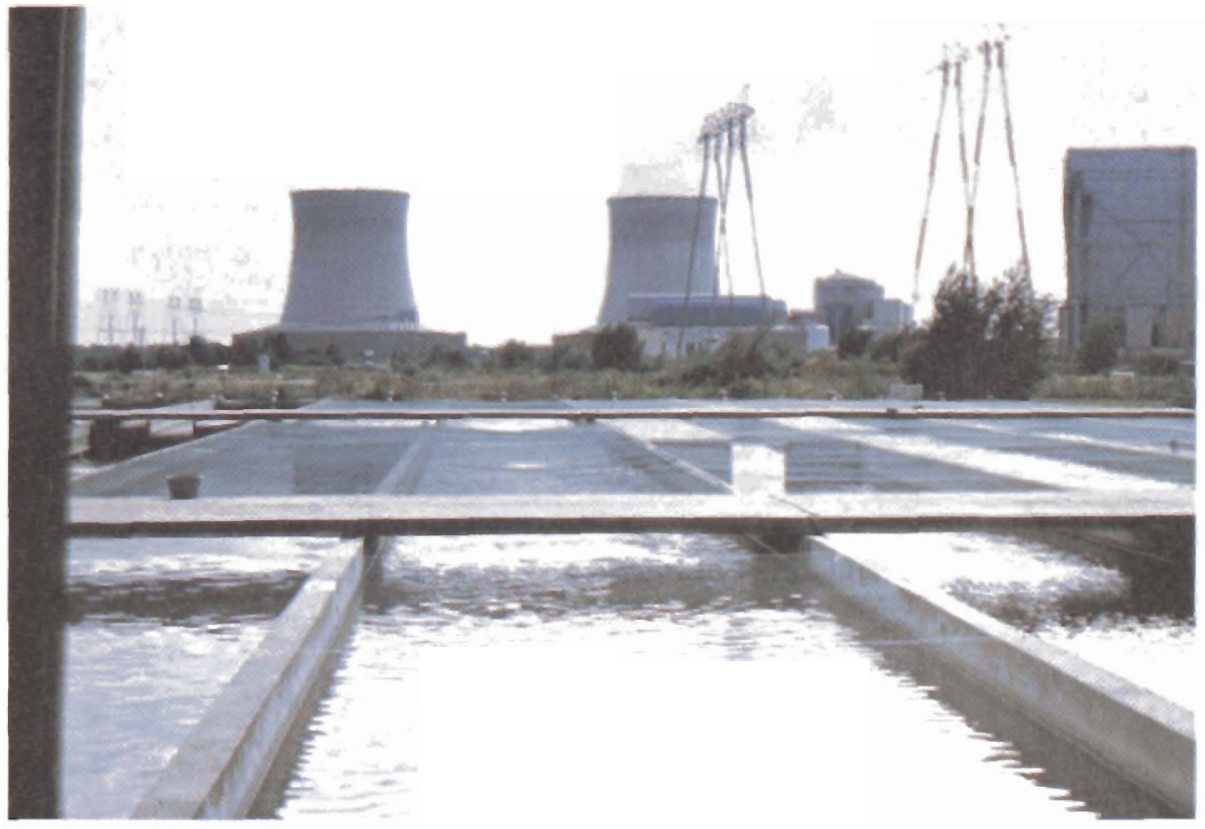

Pholo 4 - Station d'aquaculture de Saint-Laurent des Eaux (41). Vue générale des bassins extérieurs.

sur les anguilles se sont arrêtés en 1984.

Des essais ont été menés en parallèle sur les tilapias dans une partie de l'installation plus particulièrement réservée à la formation et aux essais. Ils avaient pour but la mise au point de techniques d'élevage qui seraient ensuite mises en application sur d'autres sites. Malgré des résultats probants, ces essais n'ont pas été suivis de réalisations industrielles faute de débouchés commerciaux suffisants pour cette espèce en France.

Enfin, une tentative d'élevage de poissons d'ornement a eu lieu en 1985. Malgré de bons résultats tech- niques, elle n'a pas non plus atteint la rentabilité économique. Ceci a poussé E.D.F. à fermer en 1986 ce site d'essais où de nombreuses informations sur les choix techniques à retenir avaient pu être obtenues mais qui ne convenait pas à une exploitation industrielle.

Au travers de ces expériences, E.D.F. a acquis de solides informations sur les possibilités et les limites de l'utilisation des eaux chaudes à. des fins piscicoles. A partir de 1986, une réflexion a été engagée en relation avec le Ministère de la $\mathrm{Re}$ cherche et le Ministère de l'Agriculture pour valoriser cet acquis en portant plus particulièrement les 
efforts vers la mise en place d'unités aquacoles de taille industrielle. Ainsi, après l'époque «pionnière», d'autres sites ont fait et font encore l'objet d'expérimentations.

\section{Nogent-sur-Seine}

De 1986 à 1988, un pilote expérimental d'élevage d'anguilles en circuit fermé a fonctionné à proximité du site de Nogent-sur-Seine. L'apport d'eau tiède au circuit, qui pourrait venir d'une tranche nucléaire, était simulé par une chaufferie. Ce pilote, financé par une entreprise privée et l'ANVAR, avait pour but de tester une technologie d'élevage danoise et de collecter tous les paramètres technico-économiques nécessaires au choix de la création ou non d'une unité de 300 t/an. Les essais, qui ont duré deux ans, ont montré que les risques inhérents à l'élevage en circuit fermé de ce type de poisson étaient trop importants au regard de la rentabilité escomptée. En conséquence, l'entreprise et ses co-investisseurs n'ont pas donné suite au projet.

\section{Le Blayais}

Une station expérimentale a été mise en place sur ce site en 1982 (Photo 5). Des essais ont été réalisés sur différentes espèces (bars, anguilles, daurades) qui ont permis d'af-

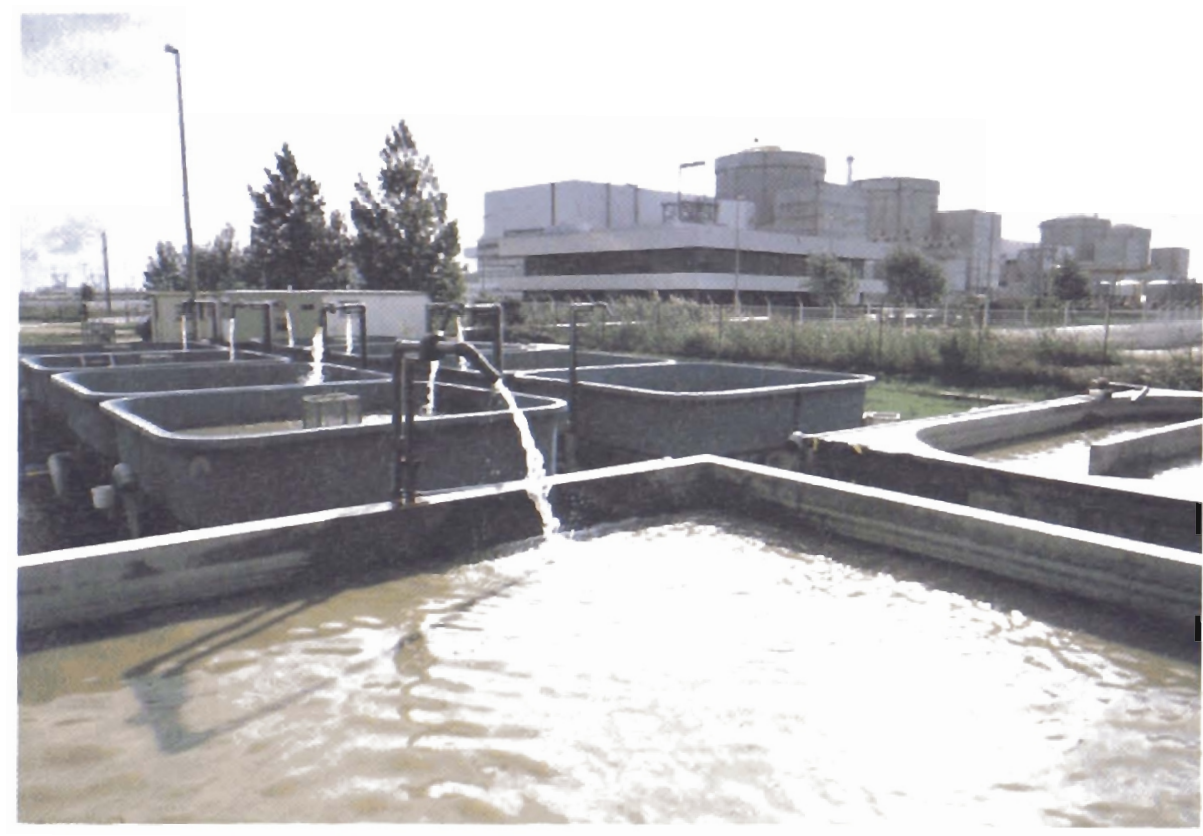

Photo 5 - Sites du Blayais (33).

Station d'expérimentations aquacoles. 
finer les résultats obtenus à Bordeaux-Ambès.

A partir de 1985, la station expérimentale a été reprise par le Syndicat Mixte du Blayais. Dès lors, des études, supervisées par un Comité d'Etudes et menées par le Syndicat des Pêcheurs professionnels de la Gironde, ont été entreprises sur le grossissement de l'esturgeon sibérien, Acipenser baeri. Les résultats de croissance se sont avérés très bons et, après deux ans d'essais, l'expérience acquise a paru suffisante pour envisager une exploitation industrielle de ce poisson sur le site. Un premier dossier de faisabilité économique pour réaliser une ferme industrielle de bars et d'esturgeons a été monté par Sepia International en 1987-1988.

Ce projet, soutenu par EDF, a été repris par les collectivités régionales et départementales, qui ont souhaité examiner les possibilités de réaliser sur le site, non pas une seule ferme aquacole mais une véritable zone capable d'abriter au moins trois fermes d'exploitation pour un potentiel de 400 à 500 tan. Nous verrons au $\S 3 \mathrm{ce}$ qu'il en est actuellement de ce projet.

La station expérimentale, reprise par Sepia International, a continué à fonctionner comme base de grossissement d'esturgeons et comme lieu de présentation de l'esturgeon sibérien jusqu'à l'été 1990. Son arrêt a été programmé pour raison de vétusté et les poissons ont été transférés sur un pilote installé dans les Landes à Arjuzanx. La commercialisation des poissons produits (la biomasse présente sur le site a dépassé 5 tonnes) a débuté en octobre 1989 avec beaucoup de succès.

\section{Cattenom}

Le site de Cattenom présente la particularité d'être assez éloigné de la Moselle et d'utiliser pour le refroidissement des eaux de purge des réfrigérants atmosphériques, une retenue artificielle de 95 ha, le lac du Mirgenbach.

Ceci offre la possibilité de développer des techniques d'élevage piscicoles différentes de celles utilisées classiquement en eaux tièdes, en mettant en place des structures flottantes sur la retenue. Ce type de valorisation a été choisi, bien que la retenue ne soit échauffée que de quelques degrés par rapport à la Moselle, compte tenu de son faible coût d'investissement. Un ponton de huit cages flottantes a été mis en place en juillet 1989 (Photo 6 et Figure 5). Les essais, sur des carpes, menés par le Laboratoire d'Ecologie de Metz, permettent de sensibiliser les pisciculteurs régionaux aux potentialités du site. Dès 1991, une extension des cages est prévue et un pilote à terre, utilisant directement les eaux tièdes, est envisagé.

Certains projets, qui ont été développés après 1986, n'ont pas abouti pour différentes raisons qui ont pu être analysées. La plupart du temps il s'agit d'une maîtrise insuffisante des techniques d'élevage ce qui laisse à penser que des projets viables pourront voir le jour lorsque les pisciculteurs domineront mieux ces problèmes. C'est le cas notamment 


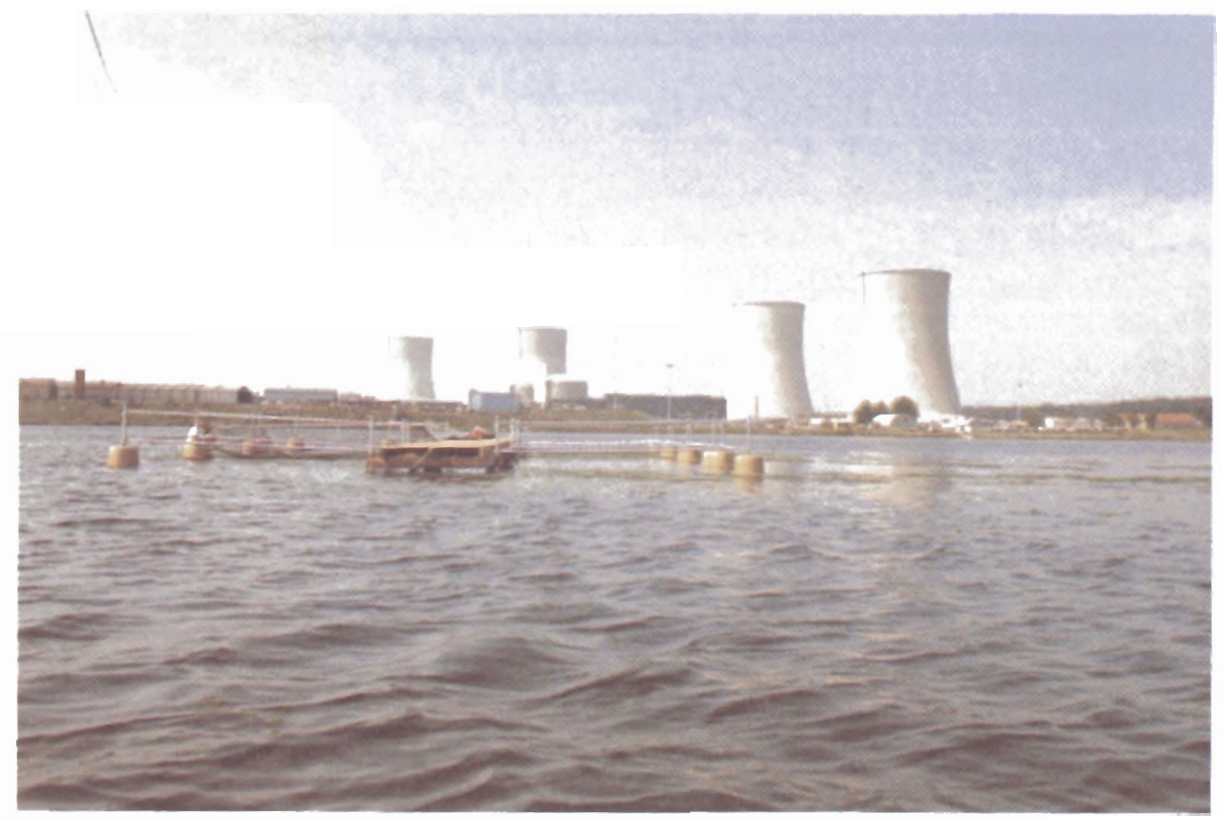

Photo 6 - Site de Cattenom (57).

Cages flottantes sur la retenue du Mirgenbach.

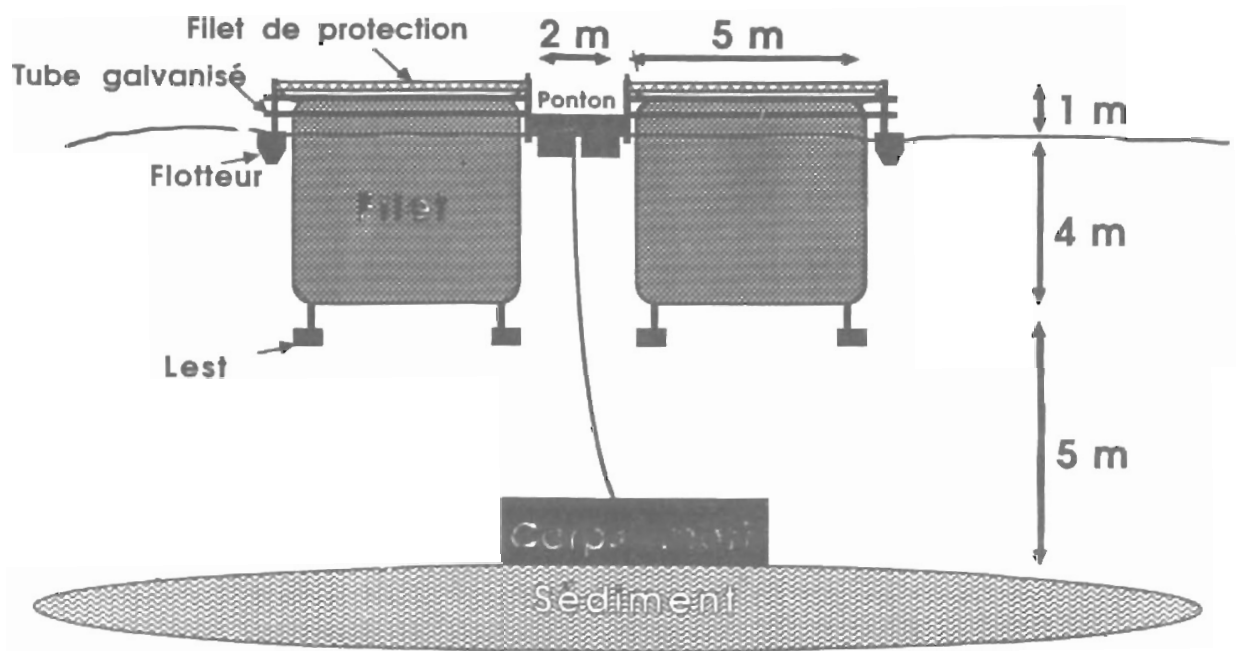

Fig. 5 - Représentation schématique des cages flottantes inștallées sur la retenue du Mirgenbach. 
de l'anguille comme à Nogent mais aussi dans une moindre mesure du silure, poisson très thermophile, pour lequel a été étudié un projet d'élevage intensif au Bugey, (projet qui également a été contrarié par des problèmes d'alimentation en eau froide, combinés à une mauvaise qualité physico-chimique de l'eau du Rhône).

Un autre point essentiel à ne pas négliger est la présence d'un marché pour le poisson élevé. Ainsi il n'a pas été possible de créer des unités d'élevage de tilapias à Cruas, comme cela avait été envisagé après les essais de St-Laurent, ces poissons n'ayant pas de débouchés sur le marché français du poisson.

\section{3. - LES RÉALISATIONS INDUSTRIELLES}

Quelques projets, trop rares encore bien sûr, se sont cependant développés et complètent maintenant l'approche expérimentale par des résultats technico-économiques en grandeur réelle.

\subsection{Site de Gravelines}

Le Centre de Production Nucléaire (CPN) de Gravelines, implanté en bordure de la Mer du Nord, pompe l'eau froide nécessaire au refroidissement des six tranches du site dans

SCHEMA D'AMENAGEMENT DE LA ZONE AQUACOLE DE GRAVELINES (59)

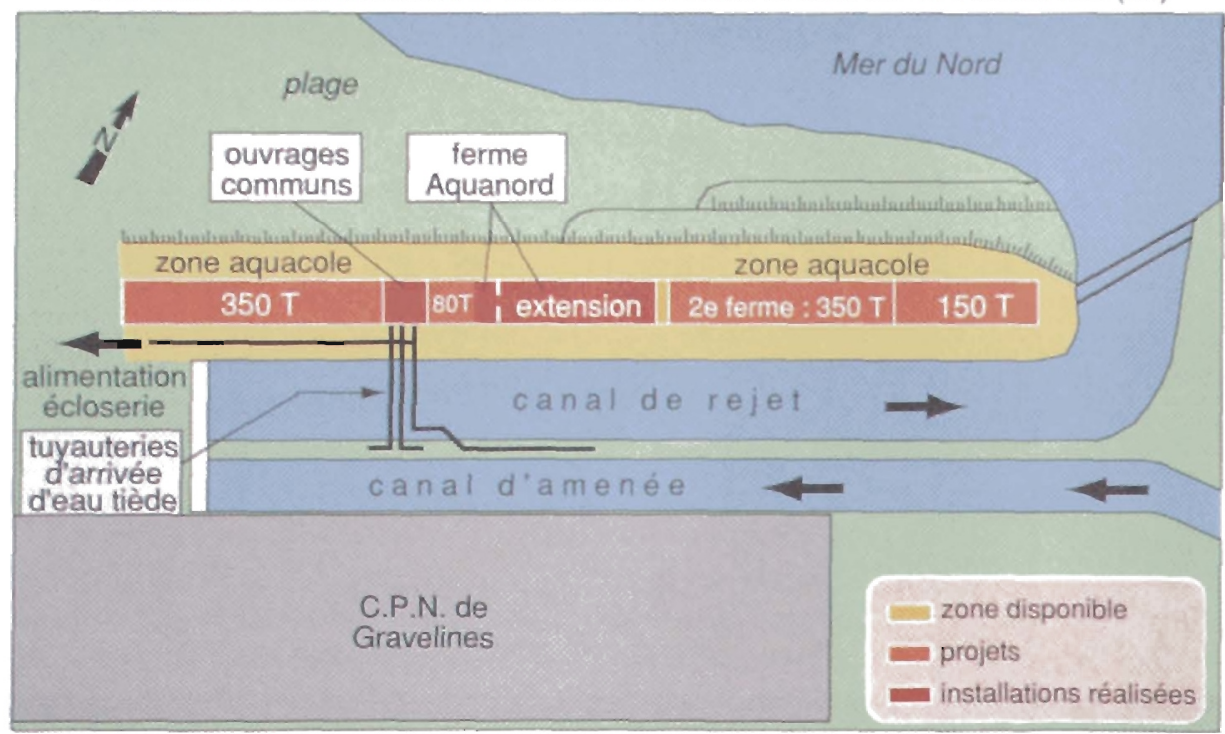


l'avant-port de Dunkerque et rejette les eaux tièdes en mer par un long canal de rejet bordé d'une bande de dunes (schéma de la photo 7).

L'intérêt d'utiliser un tel site pour l'aquaculture est très vite apparu et a conduit, dès 1982, la commune de Gravelines, le Syndicat Intercommunal d'Alimentation en eau de la Région de Dunkerque (S.I.A.E.R.D.) et Electricité de France à créer le S.E.R.A.G. (Syndicat mixte pour l'Etude d'un Réseau d'Aquaculture à Gravelines).

Pour tester la faisabilité technique et économique de l'élevage de poissons marins en eaux tièdes le SERAG a réalisé en 1983-1984:
- une ferme aquacole pilote de $3000 \mathrm{~m}^{2}$ exploitée par la coopérative maritime Aquanord.

- une station d'Essais et de Formation Aquacole, S.E.F.A., gérée par I'I.F.R.E.M.E.R.

Parallèlement, EDF a fait réaliser la mise en place de tuyauteries et de siphons qui permettront de prélever jusqu'à $13 \mathrm{~m}^{3} / \mathrm{s}$ d'eaux tièdes sur les rejets de 3 des 6 tranches. L'eau froide est prélevée dans le canal de prise d'eau. La possibilité d'opérer des mélanges pour optimiser la température d'élevage est un des avantages décisifs du site.

- La S.E.F.A. qui a fonctionné jusqu'en 1988, outre une assistance à

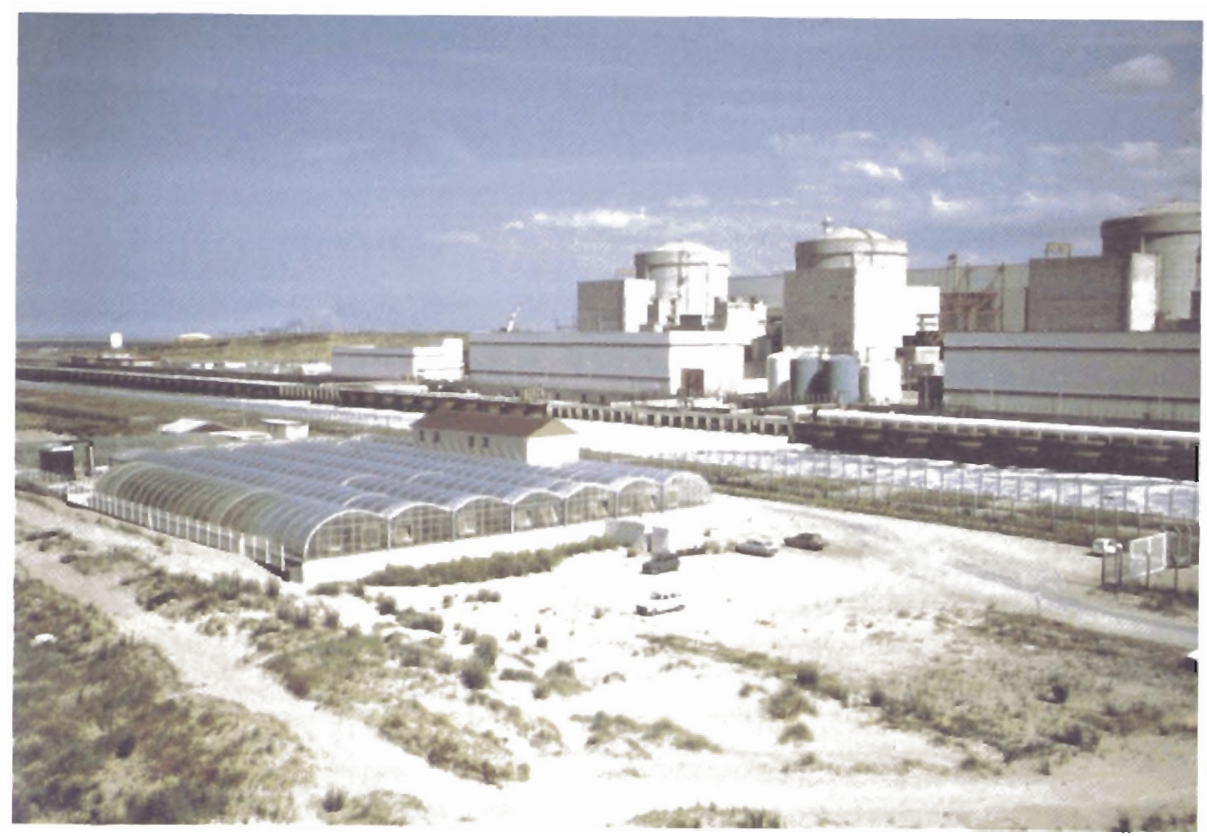

Photo 8 - Site de Gravelines (59).

Ferme de grossissement de bars. Aquanord SA. 


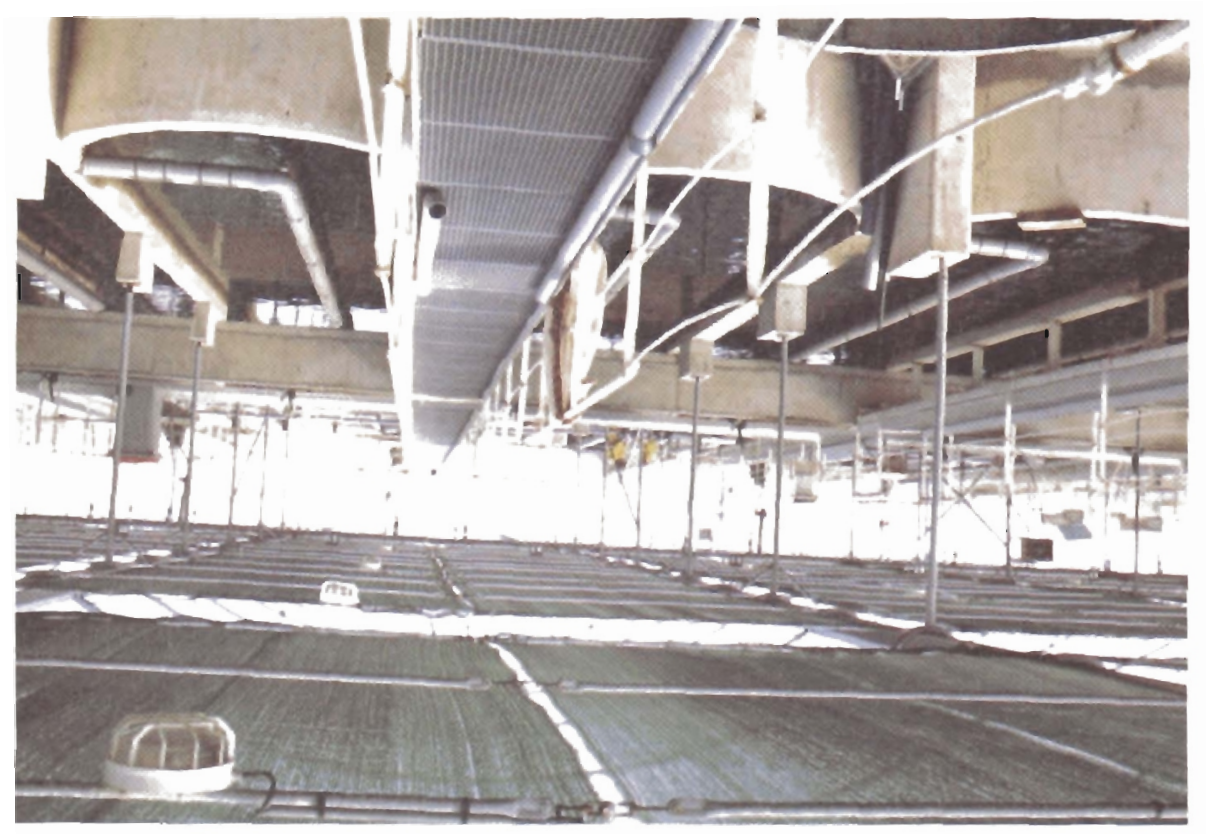

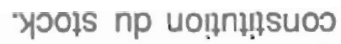

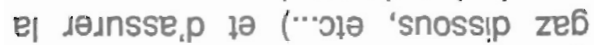

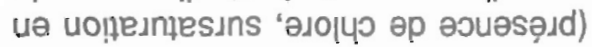

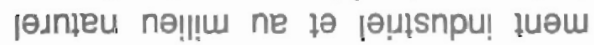

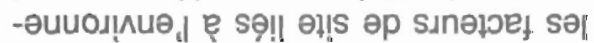

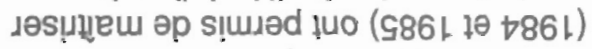

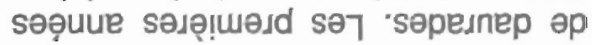

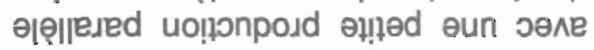

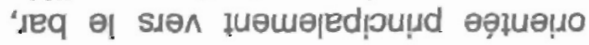

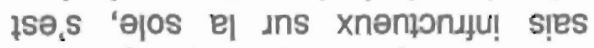

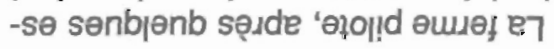

-s!low 91 e

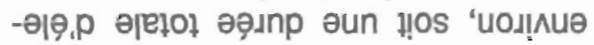

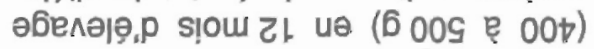

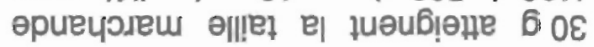

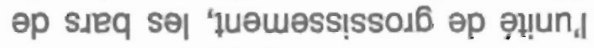

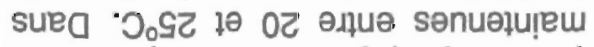

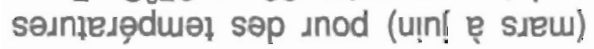

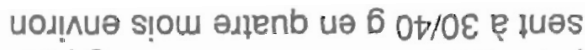

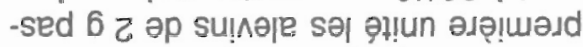

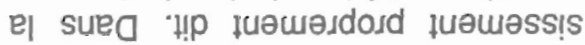

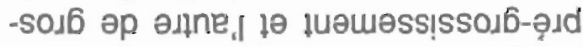
әp әun “søฺ!un-snos xnәp puәıduos

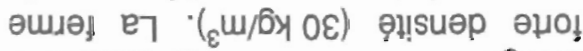

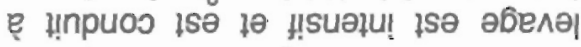

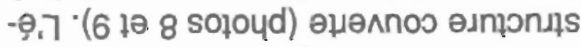
snos әредuә !ored ę uolpq su!sseq

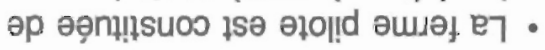

$\cdots$ ".

'sloqu! 'sәpednep 'sıeq 'sәщәләдо

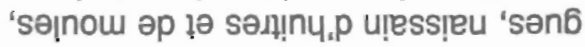
-

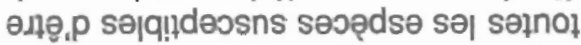

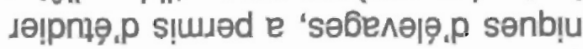

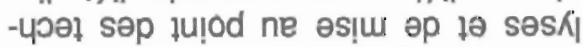

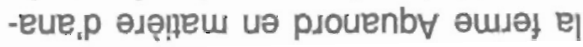


Les premières ventes de bars portion (environ $400 \mathrm{~g}$ ) ont été réalisées en 1984 ( 5 tonnes) et ont progressé rapidement pour atteindre 65 tonnes en 1988. Ce succès a conduit les partenaires à transformer Aquanord en société anonyme en 1988 et à en faire la première ferme aquacole industrielle implantée sur le site. La gestion en est confiée à Sepia International. La production est passée à 83 tonnes en 1989 et 77 tonnes en 1990, années aux conditions météorologiques favorables. II faut rappeler que le tonnage annuel initialement prévu sur Aquanord est de 65 tonnes (Brunel et al., 1989].

Pour poursuivre le développement aquacole du site, une écloserie de poissons marins a été installée, à proximité immédiate de la zone aquacole, en 1987. La S.E.M., Ecloserie Marine de Gravelines, également gérée par Sepia International, a été implantée sur un terrain de $20000 \mathrm{~m}^{2}$. Alimentée en eau tiède et en eau de nappe phréatique, elle utilise des techniques sophistiquées de traitement des eaux (pompe à chaleur, recyclage avec biofiltration et stérilisation UV, etc...) qui lui permettent une maitrise totale du milieu d'élevage.

Les installations de l'écloserie comprenaient, en première phase de développement, outre des bâtiments d'usage général et un bâtiment technique d'alimentation et de traitement des eaux, une unité de production de proies vivantes (artémias, rotifères, nécessaires pour les jeunes stades qu'il n'est pas possible de nourrir avec des aliments artificiels - granulés - comme les poissons en phase de grossissement), une unité de stockage et de conditionnement des géniteurs et deux unités d'élevage identiques dimensionnées chacune pour une production nominale de 500000 alevins de $2 \mathrm{~g}$ par an (photo 10 ).

Dès la première année d'exploitation, cette installation a dépassé sa production nominale pour atteindre les deux millions d'alevins de bars en 1989. En 1990, une première extension des installations a été réalisée avec un agrandissement de l'unité de production de proies pour pouvoir cultiver des algues et l'ajout d'une serre d'élevage larvaire. Ceci permet à l'Ecloserie Marine de diversifier ses productions avec une capacité de production de 200000 daurades et 300000 turbots par an.

En 1991/92, les travaux d'agrandissement vont se poursuivre pour atteindre la phase finale de développement de cette écloserie, qui pourra alors produire environ 2 millions de bars de plus, soit un total de 4 millions, et qui possèdera une unité de recherche-développement sur le développement larvaire des poissons et crustacés d'eaux marines tempérées et chaudes.

La plupart des alevins produits à l'Ecloserie Marine de Gravelines est destinée à poursuivre sa croissance sur la zone aquacole de Gravelines, dont le développement a débuté en 1988 après une série d'études menées par le SERAG, précisant les solutions techniques appropriées au site 


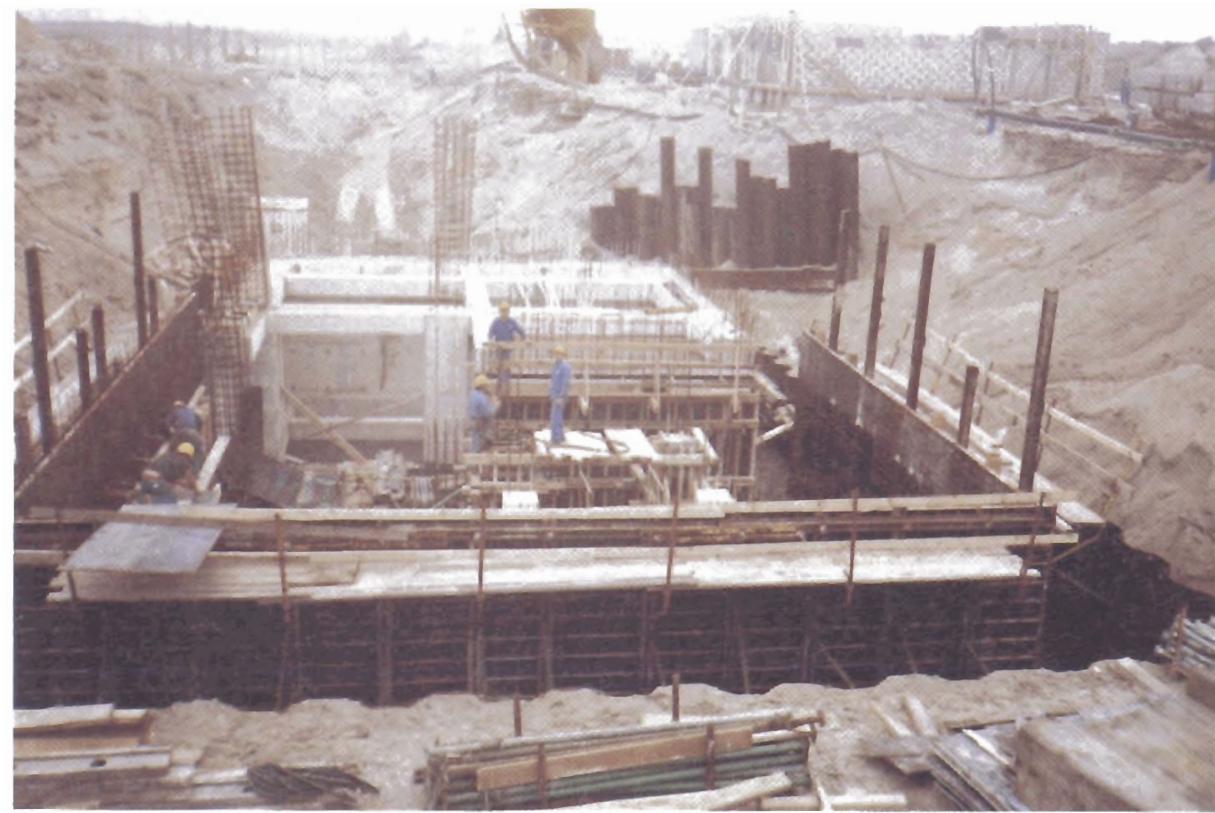

Photo 11 - Création du complexe de gestion des eaux par la S.E.M. Gravelines Aquaculture.

ouvrages communs et devraient être terminés au printemps 1991. Les bassins, du même type que ceux mis en place dans la ferme pilote mais bénéficiant de modifications liées à l'expérience acquise, sont regroupés dans un bâtiment couvert de $216 \mathrm{~m}$ de long (photo 12).

- Une deuxième ferme, d'une capacité de 350 T/an, produisant des bars mais aussi peut-être des turbots, est en cours de montage financier. Les travaux de construction devraient débuter fin 1991.

Ces deux réalisations, assistées de l'Ecloserie Marine de Gravelines, feront du Centre Aquacole de Grave- lines le plus grand site français d'élevage de poissons marins. Mais les ambitions ne s'arrêtent pas là. En raison des débits d'eau et des terrains encore disponibles (photo 7), la capacité de production de l'ensemble du site aquacole pourrait atteindre 1200 à 1400 tonnes par an de produits de l'aquaculture.

\subsection{Site du Blayais}

Comme nous l'avons indiqué précédemment, il existait une volonté de développer à proximité du Centre de Production Nucléaire du Blayais, situé sur la Gironde, une zone aquacole 


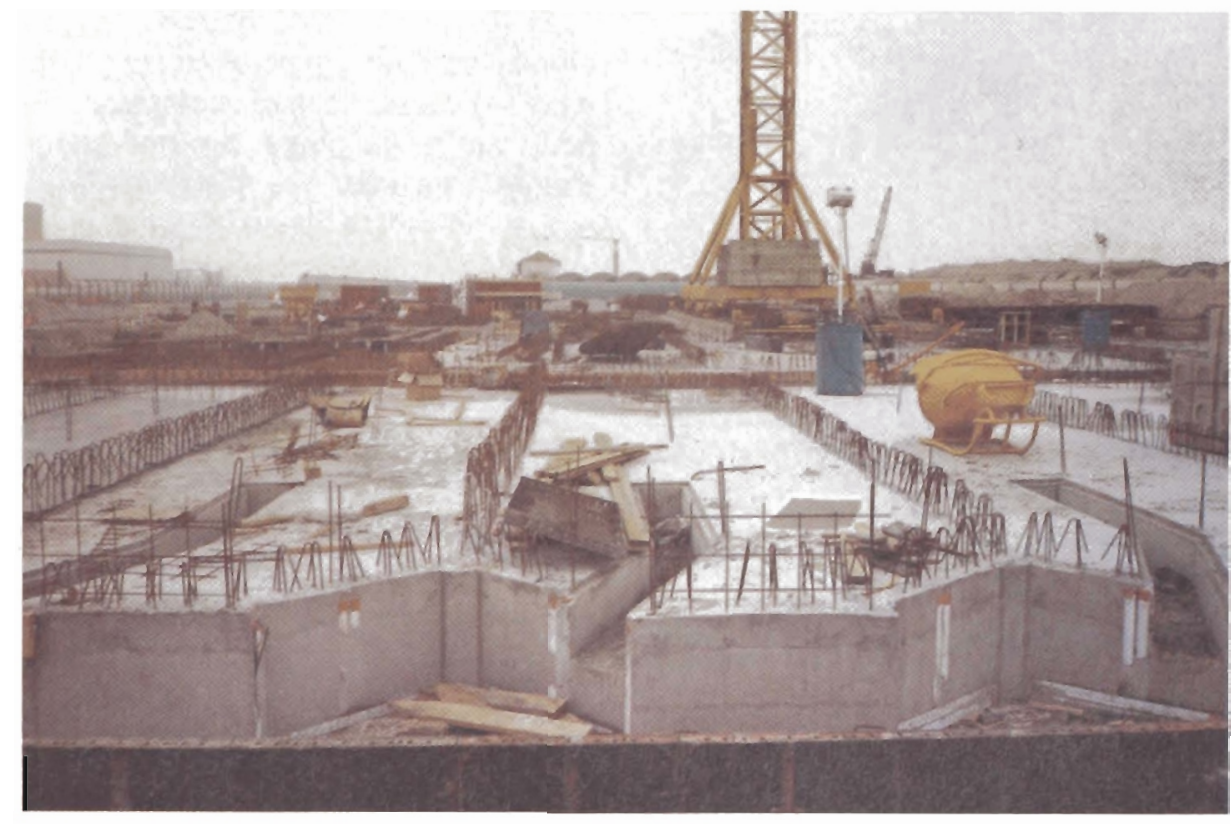

Photo 12 - Travaux d'extension d'Aquanord pour porter sa capacité de production à 300 /an. Création d'un bâtiment de $216 \mathrm{~m}$ de long.

qui, un peu comme à Gravelines, permette, par l'installation de plusieurs fermes aquacoles, de créer un pôle régional de compétences et d'emplois, et de rentabiliser la mise en place onéreuse d'un réseau d'alimentation en eau tiède.

Des études ont été entreprises par le Conseil Général de la Gironde et le Syndicat Mixte du Blayais pour créer une zone aquacole au Sud de la centrale et pour y implanter plusieurs professionnels de l'aquaculture.

Après deux ans de recherches et de consultations, le Conseil Général de la Gironde est cependant arrivé à la conclusion fin 1990 que le projet initial de produire 400 à 500 T/an de bars et d'esturgeons sur une zone située au Sud de la centrale était irréalisable, essentiellement du fait du coût des travaux à entreprendre pour stabiliser les sols (marais). Des solutions de remplacement ont été recherchées car le site permet, notamment pour l'esturgeon, d'obtenir des performances zootechniques très valorisantes et il eût été dommage de l'abandonner.

Actuellement, un nouveau projet, de taille plus modeste en ce qui concerne la quantité de poissons que l'on pourra produire, est à l'étude sur des terrains situés au Nord de la centrale et mieux stabilisés. 


\section{4. - LES PERSPECTIVES}

Après ce bilan, quelles sont les perspectives?

Les perspectives sont intéressantes, même si elles sont devenus plus "raisonnables" qu'il y a quelques années, où l'on imaginait volontiers chaque site nucléaire entouré de sa cohorte de serres et de bassins aquacoles en tous genres. Avec l'expérience, on est maintenant capable de déterminer techniquement et économiquement s'il est possible, compte tenu de l'implantation géographique d'un site, de sa topographie, de son mode d'alimentation en eau, du nombre de tranches, d'envisager une utilisation des eaux tièdes. On peut également formuler un avis sur le mode de développement (serres d'un certain type, aquaculture particulière...) qui conviendrait le mieux au site considéré et à son contexte économique et social. Enfin, dans le cadre d'une concertation accrue avec les partenaires locaux qui souhaitent développer de tels projets, un avis technique peut être donné sur la qualité des projets et leurs chances de réussite.

Les principaux espoirs actuels concernent les deux sites en cours de développement, Gravelines et Le Blayais. La réussite à l'échelle industrielle d'élevages intensifs en eau tiède permettra de prouver l'intérêt économique d'une telle solution, qui ne peut se faire que grâce à des équipements collectifs coûteux dont la gestion doit être rigoureuse.
En dehors de ces deux sites, des études sont en cours avec les partenaires locaux et les professionnels pour mettre en place des opérations d'aquaculture sur des sites déjà équipés de réseaux d'eau tiède desservant des installations de maraîchage ou d'horticulture, là où des terrains sont encore disponibles.

Ainsi, sur le site de Chinon, où la qualité du réseau d'eau tiède permet une alimentation en eau tiède à une température toujours supérieure à $30^{\circ} \mathrm{C}$, on examine les possibilités de créer une unité d'élevage de poissons d'ornement.

A Cruas, c'est la possibilité de réutiliser une seconde fois les eaux tièdes après le passage dans des serres de culture de tomates qui est sérieusement envisagée pour alimenter une ferme d'élevage d'esturgeons.

Enfin, les dernières possibilités, qui ne sont pas négligées, sont de créer de nouveaux réseaux d'eau tiède sur des sites qui n'en sont pas encore équipés.

Cette possibilité est actuellement à l'étude pour le site de Cattenom, qui disposera à terme de quatre tranches équipées de réfrigérants atmosphériques. Sur ce site, la volonté des communes limitrophes regroupées au sein du District de Cattenom a toujours été de réaliser autour du CPN une zone d'activités et de loisirs, qui crée des emplois mais aussi qui contribue à l'intégration du site dans l'environnement. Depuis deux ans, des réflexions ont été entreprises pour définir les activités qui pourraient 
se développer, soit autour et sur la retenue du Mirgenbach, soit en utilisant les eaux tièdes. De très nombreuses voies sont expiorées : piscine et centre de loisirs utilisant en partie la retenue pour des activités nautiques, serres, technologies algales à partir d'une espèce d'algue verte filamenteuse qui se développe préférentiellement dans la retenue, aquaculture intensive à terre et en cages flottantes, pré-séchage du bois, etc...

A la demande du District du Cattenom, la Direction de l'Equipement d'EDF a réalisé en 1990 une étude préliminaire d'aménagement du site de Caltenom. Cette étude présente les différents axes de développement possibles du site et devrait permettre au District de choisir la ou les activités qui seront mise en œuvre prioritairement. Cette étude est typique de l'apport qu'EDF, avec l'expérience accumulée dans le domaine de l'utjlisation des eaux tièdes, est aujourd'hui à même de fournir aux collectivités locales et aux professionnels qui souhaitent valoriser les eaux tièdes disponibles auprès des C.P.N.

\section{CONCLUSIONS}

L'aquaculture en eau tiède est une activité spécifique qui est née de la possibilité de disposer de grandes quantités d'eau faiblement échauffée et rejetée dans le milieu naturel dont elle est issue.
Encore fallait-il pouvoir capter ces volumes d'eau dans des conditions économiquement rentables et trouver des espèces et des méthodes d'élevage adaptées. Après de nombreuses expérimentations, l'expérience acquise est aujourd'hui suffisamment importante pour conduire à la mise en place d'unités de production de taille industrielle.

Tous les sites ne présentent pas les mêmes possibilités d'utilisation d'eau tiède et les espèces de poissons à élever en eaux tièdes doivent être soigneusement choisies. La croissance en eau tiède doit apporter un gain économique appréciable compensant les charges liées aux ouvrages complémentaires nécessités par l'utilisation de l'eau tiède (canalisations essentiellement). On estime qu'en moyenne le coût total de la fourniture de l'eau est de 8 à 13 francs par kilogramme de poisson produit, ce qui représente autant dans le prix de revient que les charges de nourriture. Ceci explique que les installations qui se mettent en place concernent des espèces - bars, daurades, turbots - dites de haut de gamme.

On peut espérer dans un proche avenir voir se poursuivre à Gravelines et se développer sur d'autres sites, notamment au Blayais, une aquaculture en eau tiède performante et rentable. Le rôle qu'EDF souhaite jouer dans ce développement ne se limite pas à la fourniture gratuite de l'eau tiède, elle peut et veut être également un partenaire technique en apportant aux investisseurs potentiels et aux 
collectivités locales qui le souhaitent le savoir acquis sur ce sujet depuis plus de quinze ans.

Travail réalisé avec la collaboration de M. HERANDE, Chargé de Mission Chaleur auprès de la Direction Générale d'Electricité de France

\section{RÉFÉRENCES}

Brunel G. et Fuzeau P., 1989. L'aquaculture en eaux réchauffées. Aquaculture, Volume 2. Technique et Documentation - Lavoisier : 889-910.

Duret J. et Proteau J.P., 1989. Diversification de l'aquaculture par utilisation des eaux réchautfées et des eaux souterraines. Publication de l'Association pour le Développement de l'Aquaculture, $20: 41-50$.

E.D.F., Direction de l'Equipement, 1990. Eaux tièdes, mode d'emploi. Plaquette de présentation $\mathrm{N}^{\circ}$ édition EG 5063/A.

Hussenot J. et Leclercq D., 1986. Sursaturation des gaz dissous, un phénomène souvent mal connu en aquaculture. Aqua-Revue, 11:27-31.

Philippart J.C., Melard C.H. et Ducarme C., 1989. Utilisation des rejets thermiques des centrales électronucléaires de Tihange pour l'élevage des poissons. Actes du Séminaire International Valorisation des rejets thermiques des centrales électriques. CCE. Mars 89 EUR 12423 EN/FR : 36-47. 